\title{
On Continuous Programming with Support Functions
}

\author{
Iqbal Husain ${ }^{1}$, Santosh K. Shrivastav ${ }^{1}$, Abdul Raoof Shah ${ }^{2}$ \\ ${ }^{1}$ Department of Mathematics, Jaypee University of Engineering and Technology, Guna, India \\ ${ }^{2}$ Department of Statistics, University of Kashmir, Srinagar, India \\ Email: ihusain11@yahoo.com
}

Received June 12, 2013; revised July 12, 2013; accetped July 19, 2013

Copyright (C) 2013 Iqbal Husain et al. This is an open access article distributed under the Creative Commons Attribution License, which permits unrestricted use, distribution, and reproduction in any medium, provided the original work is properly cited.

\begin{abstract}
A second-order Mond-Weir type dual problem is formulated for a class of continuous programming problems in which both objective and constraint functions contain support functions; hence it is nondifferentiable. Under second-order strict pseudoinvexity, second-order pseudoinvexity and second-order quasi-invexity assumptions on functionals, weak, strong, strict converse and converse duality theorems are established for this pair of dual continuous programming problems. Special cases are deduced and a pair of dual continuous problems with natural boundary values is constructed. A close relationship between the duality results of our problems and those of the corresponding (static) nonlinear programming problem with support functions is briefly outlined.
\end{abstract}

Keywords: Continuous Programming; Second-Order Generalized Invexity; Second-Order Duality; Nonlinear Programming; Support Functions; Natural Boundary Values

\section{Introduction}

Chen [1] was the first to identify second-order dual formulated for a constrained variational problem and established various duality results under an involved invexitylike assumptions. Husain et al. [2] have presented MondWeir type second-order duality for the problem of [1] and by introducing continuous-time version of secondorder invexity and generalized second-order invexity, validated various duality results. Subsequently, for a class of nondifferentiable continuous programming problems, Husain and Masoodi [3] studied Wolfe type second-order duality while Husain and Srivastava [4] investigated MondWeir type second-order duality. Recently, in the spirit of Mangasarian [5], Husain and Masoodi [6] studied Wolfe type second-order duality for a continuous programming problem having support functions appearing in the integrand of the functional as well as in the constraint functions under second-order invexity and second-order pseudoinvexity conditions. They also incorporated a pair of second-order dual variational problems with natural boundary values rather than fixed end points and indicated their close relationship with those of corresponding (static) second-order duality results established for nonlinear programming problem with support functions, considered by Husain et al. [7]. The popularity of this type of nondifferentiable continuous programming problems seems to originate from the fact that, even though the objective function and/or constraint functions are non-smooth, a simple representation of the dual problem may be written. The theory of non-smooth mathematical programming deals with more general type of functions by means of generalized subdifferentials. However, square root of positive semi-definite quadratic form and support functions are amongst few cases of the nondifferentiable functions for which one can write down the subdifferentials explicitly.

In this paper, we formulate Mond-Weir type secondorder dual to the continuous programming containing support functions in order to further weaken the secondorder generalized invexity of [6]. Usual duality theorems for this pair of Mond-Weir type second-order dual continuous programming problems are validated under generalized second-order invexity assumptions. Special cases are derived. Further, a pair of Mond-Weir second-order dual variational problems with natural boundary values rather than fixed end points is presented and the proofs of the duality theorems are claimed to follow analogously. It is also pointed out that our second-order duality results can be considered as dynamic generalizations of corresponding (Static) second-order duality results established for nonlinear programming problem with support functions considered by Husain et al. [7]. 


\section{Pre-Requisites}

Let $I=[a, b]$ be a real interval $\phi: I \times R^{n} \times R^{n} \rightarrow R$ and $\psi: I \times R^{n} \times R^{n} \rightarrow R^{m}$ be twice continuously differentiable functions. In order to consider $\phi(t, x(t), \dot{x}(t))$, where $x: I \rightarrow R^{n}$ is differentiable with derivative $\dot{x}$ denoted by $\phi_{x}$ and $\phi_{\dot{x}}$ the first order derivative of $\phi$ with respect to $x(t)$ and $\dot{x}(t)$ respectively, that is,

$$
\phi_{x}=\left(\frac{\partial \phi}{\partial x^{1}}, \frac{\partial \phi}{\partial x^{2}}, \cdots, \frac{\partial \phi}{\partial x^{n}}\right)^{\mathrm{T}}, \phi_{\dot{x}}=\left(\frac{\partial \phi}{\partial \dot{x}^{1}}, \frac{\partial \phi}{\partial \dot{x}^{2}}, \cdots, \frac{\partial \phi}{\partial \dot{x}^{n}}\right)^{\mathrm{T}} .
$$

Denote by $\phi_{x x}$ the $n \times n$ Hessian matrix of $\phi$ and $\psi_{x}$ the $m \times n$ Jacobian matrix respectively, that is, with respect to $x(t)$, that is,

$\phi_{x x}=\left(\frac{\partial^{2} \phi}{\partial x^{i} \partial x^{j}}\right), i, j=1,2, \cdots, n \quad \psi_{x}$ the $m \times n$ Jacobian matrix.

$$
\psi_{x}=\left(\begin{array}{cccc}
\frac{\partial \psi^{1}}{\partial x^{1}} & \frac{\partial \psi^{1}}{\partial x^{2}} & \cdots & \frac{\partial \psi^{1}}{\partial x^{n}} \\
\frac{\partial \psi^{2}}{\partial x^{1}} & \frac{\partial \psi^{2}}{\partial x^{2}} & \cdots & \frac{\partial \psi^{2}}{\partial x^{n}} \\
\vdots & \vdots & \ddots & \vdots \\
\frac{\partial \psi^{m}}{\partial x^{1}} & \frac{\partial \psi^{m}}{\partial x^{2}} & \cdots & \frac{\partial \psi^{m}}{\partial x^{n}}
\end{array}\right)_{m \times n}
$$

The symbols $\phi_{\dot{x}}, \phi_{\dot{x} x}, \phi_{x \dot{x}}$ and $\psi_{\dot{x}}$ have analogous representations. Designate by $\mathrm{X}$ the space of piecewise smooth functions $x: I \rightarrow R^{n}$, with the norm $\|x\|=\|x\|_{\infty}+\|D x\|_{\infty}$ where the differentiation operator $D$ is given by $u=D x \Leftrightarrow x(t)=\int_{a}^{t} u(s) \mathrm{d} s$, Thus $\frac{\mathrm{d}}{\mathrm{d} t}=D$ except at discontinuities.

We incorporate the following definitions which needed in the subsequent analysis:

\section{Definition1. (Second-Order Invex):}

If there exists a vector function $\eta=\eta(t, x, \bar{x}) \in R^{n}$ where $\eta: I \times R^{n} \times R^{n} \rightarrow R^{n}$ and with $\eta=0$ at $t=a$ and $t=b$ such that for a scalar function $\phi(t, x, \dot{x})$ the functional
satisfies

$$
\begin{aligned}
& \int_{I} \phi(t, x, \dot{x}) \mathrm{d} t-\int_{I}\left\{\phi(t, \bar{x}, \dot{\bar{x}})-\frac{1}{2} p^{\mathrm{T}}(t) G p(t)\right\} \mathrm{d} t \\
& \geq \int_{I}\left\{\eta^{\mathrm{T}} \phi_{x}(t, \bar{x}, \dot{\bar{x}})+(D \eta)^{\mathrm{T}} \phi_{\dot{x}}(t, \bar{x}, \dot{\bar{x}})+\eta^{\mathrm{T}} G p(t)\right\} \mathrm{d} t .
\end{aligned}
$$

Then $\int_{I} \phi(t, x, \dot{x}) \mathrm{d} t$ is second-order invex with respect to $\eta$ where $G=\phi_{x x}-2 D \phi_{x \dot{x}}+D^{2} \phi_{\dot{x} \dot{x}}-D^{3} \phi_{\ddot{x} \ddot{x}}$, $p \in C\left(I, R^{n}\right)$, the space of $n$-dimensional continuous vector functions.
Definition 2. (Second-Order Pseudoinvex): If the functional $\int_{I} \phi(t, x, \dot{x}) \mathrm{d} t$ satisfies

$$
\begin{aligned}
& \int_{I}\left\{\eta^{\mathrm{T}} \phi_{x}+(D \eta)^{\mathrm{T}} \phi_{\dot{x}}+\eta^{\mathrm{T}} G p(t) p(t)\right\} \mathrm{d} t \geq 0 \\
& \Rightarrow \int_{I} \phi(t, x, \dot{x}) \mathrm{d} t \geq \int_{I}\left\{\phi(t, \bar{x}, \dot{\bar{x}})-\frac{1}{2} p(t)^{T} G p(t)\right\} \mathrm{d} t .
\end{aligned}
$$

Then $\int_{I} \phi(t, x, \dot{x}) \mathrm{d} t$ is said to be second-order pseudoinvex with respect to $\eta$.

Definition 3. (Second-Order Strictly Pseudoinvex): If the functional $\int_{I} \phi(t, x, \dot{x}) \mathrm{d} t$ satisfies

$$
\begin{aligned}
& \int_{I}\left\{\eta^{\mathrm{T}} \phi_{x}+(D \eta)^{\mathrm{T}} \phi_{\dot{x}}+\eta^{\mathrm{T}} G p(t)\right\} \mathrm{d} t \geq 0 \\
& \Rightarrow \int_{I} \phi(t, x, \dot{x}) \mathrm{d} t>\int_{I}\left\{\phi(t, \bar{x}, \dot{\bar{x}})-\frac{1}{2} p(t)^{\mathrm{T}} G p(t)\right\} \mathrm{d} t,
\end{aligned}
$$

then $\int_{I} \phi(t, x, \dot{x}) \mathrm{d} t$ is said to be second-order strictly pseudoinvex with respect to $\eta$.

Definition 4. (Second-Order Quasi-invex):

If the functional $\int_{I} \phi(t, x, \dot{x}) \mathrm{d} t$ satisfies

$$
\begin{aligned}
& \int_{I} \phi(t, x, \dot{x}) \mathrm{d} t \leq \int_{I}\left\{\phi(t, \bar{x}, \dot{\bar{x}})-\frac{1}{2} p(t)^{\mathrm{T}} G p(t)\right\} \mathrm{d} t \\
& \Rightarrow \int_{I}\left\{\eta^{\mathrm{T}} \phi_{x}+(D \eta)^{\mathrm{T}} \phi_{\dot{x}}+\eta^{\mathrm{T}} G(t) p(t)\right\} \mathrm{d} t \leq 0 .
\end{aligned}
$$

Then $\int_{I} \phi(t, x, \dot{x}) \mathrm{d} t$ is said to be second-order quasiinvex with respect to $\eta$.

Consider the following nondifferentiable continuous programming problem with support functions treated by Husain and Jabeen [8]:

$$
\text { (CP): Minimize } \int_{I}\{f(t, x, \dot{x})+S(x(t) \mid K)\} \mathrm{d} t
$$

subject to

$$
\begin{gathered}
x(a)=0=x(b), \\
g^{j}(t, x, \dot{x})+S\left(x(t) \mid C^{j}\right) \leq 0, j=1,2, \cdots, m, t \in I,
\end{gathered}
$$

where $f$ and $g$ are continuously differentiable and each $C^{j},(j=1,2, \cdots, m)$ is a compact convex set in $R^{n}$.

Husain and Jabeen [8] derived the following optimality condition for (CP):

Lemma 1. (Fritz-John Necessary Optimality Conditions):

If the problem (CP) attains a minimum at $x=\bar{X} \in X$ then there exist $r \in R$ and piecewise smooth functions $\bar{y}: I \rightarrow R^{m}$ with $\bar{y}(t)=\left(\bar{y}^{1}(t), \bar{y}^{2}(t), \cdots, \bar{y}^{m}(t)\right)$, 
$\bar{Z}: I \rightarrow R^{n}$ and $w^{j}: I \rightarrow R^{n}, \quad j=1,2, \cdots, m$, such that

$$
\begin{gathered}
r\left[f_{x}(t, \bar{x}, \dot{\bar{x}})+\bar{z}(t)\right]+\sum_{j=1}^{m} \bar{y}^{j}(t)\left[g_{x}^{j}(t, \bar{x}, \dot{\bar{x}})+\bar{w}^{j}(t)\right] \\
=D\left[r f_{\dot{x}}(t, \bar{x}, \dot{\bar{x}})+\bar{y}(t)^{\mathrm{T}} g_{\dot{x}}(t, \bar{x}, \dot{\bar{x}})\right], t \in I \\
\sum_{j=1}^{m} \bar{y}^{j}(t)\left[g^{j}(t, \bar{x}, \dot{\bar{x}})+\bar{x}(t)^{\mathrm{T}} \bar{w}^{j}(t)\right]=0, t \in I \\
\bar{x}(t)^{\mathrm{T}} \bar{z}(t)=S(\bar{x}(t) \mid K), t \in I \\
\bar{x}(t)^{\mathrm{T}} \bar{w}^{j}(t)=S\left(\bar{x}(t) \mid C^{j}\right), j=1,2, \cdots, m, t \in I \\
\bar{z}(t) \in K, w^{j}(t) \in C^{j}, j=1,2, \cdots, m, t \in I \\
(r, \bar{y}(t)) \geq 0, t \in I \\
(r, \bar{y}(t)) \neq 0, t \in I
\end{gathered}
$$

The minimum $\bar{x}(t)$ of (CP) may be described as normal if $\bar{r}=1$, so that the Fritz John optimality conditions reduce to Karush-Kuhn-Tucker optimality conditions. It suffices for $\bar{r}=1$ that Slater's [8] condition holds at $\bar{x}(t)$.

Now we review some well known facts about a support function for easy reference.

Let $K$ be a compact set in $R^{n}$, then the support function of $K$ is defined by

$$
S(x(t) K)=\max \left\{x(t)^{\mathrm{T}} v(t): v(t) \in K, t \in I\right\} .
$$

A support function, being convex everywhere finite, has a subdifferential in the sense of convex analysis i.e., there exist $z(t) \in R^{n}, t \in I$ such that

$$
S(y(t) \mid K)-S(x(t) \mid K) \geq(y(t)-x(t))^{\mathrm{T}} z(t) .
$$

From [9], the subdifferential of $S(x(t) \mid K)$ is given by $\partial S(x(t) \mid K)=\left\{z(t) \in K, t \in I\right.$ such that $\left.\mid x(t)^{\mathrm{T}} z(t)=S(x(t) \mid K)\right\}$.

For any set $\Gamma \subset R^{n}$, the normal cone to $\Gamma$ at a point $x(t) \in \Gamma$ is defined by

$N_{\Gamma}(x(t))=\left\{y(t) \in R^{n} \mid y(t)(z(t)-x(t)) \leq 0, z(t) \in \Gamma\right\}$

It can be verified that for a compact convex set $C$, $y(t) \in N_{C}(x(t))$ if and only if

$$
S(y(t) \mid C)=x(t)^{\mathrm{T}} y(t), t \in I .
$$

\section{Mond-Weir Type Second-Order Duality}

In this section, we present the following problem as the Mond-Weir type dual to (CP) and validate usual duality theorems:

(M-WCD):

$$
\operatorname{Maximize} \int_{I}\left(f(t, u, \dot{u})+u(t)^{\mathrm{T}} z(t)-\frac{1}{2} p(t)^{\mathrm{T}} F P(t)\right) \mathrm{d} t
$$

subject to

$$
\begin{gathered}
u(a)=0=u(b) \\
f_{u}+z(t)+\sum_{j=1}^{m} y^{j}(t)\left(g_{u}^{j}+w^{j}(t)\right) \\
-D\left(f_{\dot{u}}+y(t)^{\mathrm{T}} g_{\dot{u}}\right)+(F+G) p(t)=0 \\
\int_{I}\left(\sum_{j=1}^{m} y^{j}(t)\left(g^{j}+u(t)^{\mathrm{T}} \omega^{j}(t)\right)-\frac{1}{2} p^{\mathrm{T}}(t) G p(t)\right) \mathrm{d} t \geq 0 \text { (5) } \\
z(t) \in K, w^{j}(t) \in C^{j}, t \in I, j=1,2, \cdots, m \\
y(t) \geq 0, t \in I
\end{gathered}
$$

where

1) $p(t) \in R^{n}, t \in I$

2) $F=f_{\text {ии }}-2 D f_{\text {ий }}+D^{2} f_{\dot{u} \dot{u}}-D^{3} f_{\ddot{u} \ddot{u}}, t \in I$

3) $G=y(t)^{\mathrm{T}} g_{u u}-2 D\left(y(t)^{\mathrm{T}} g_{\dot{u}}\right)_{u}$

$$
+D^{2}\left(y(t)^{\mathrm{T}} g_{\dot{u} \dot{u}}\right)-D^{3}\left(y(t)^{\mathrm{T}} g_{\dot{u} u ̈}\right)
$$

Theorem 1. (Weak Duality): Let $x(t) \in X$ be feasible solution of (CP) and

$$
\left(u(t), y(t), z(t), w^{1}(t), w^{2}(t), \cdots, w^{m}(t), p(t)\right)
$$

be feasible for (M-WCD). Assume that for all feasible

$$
\left(x(t), u(t), y(t), z(t), w^{1}(t), w^{2}(t), \cdots, w^{m}(t)\right)
$$

and with respect to vector function $\eta=\eta(t, x, u)$,

1) $\int_{I}\left(f(t, . .)+.(.)^{\mathrm{T}} z(t)\right) \mathrm{d} t$ is second-order pseudoinvex and

2) $\int_{I} \sum_{j=1}^{m}\left(y^{j}(t) g^{j}(t, . . .)^{\mathrm{T}}+(.)^{\mathrm{T}} w^{j}(t)\right) \mathrm{d} t$ is second-order quasi-invex.

Then,

$$
\inf (\mathrm{CP}) \geq \sup (\mathrm{M}-\mathrm{WCD}) \text {. }
$$

Proof: Since $x(t)$ is feasible for (CP) and

$$
\left(u(t), y(t), z(t), w^{1}(t), w^{2}(t), \cdots, w^{m}(t), p(t)\right)
$$

is feasible of (M-WCD), we have

$$
\begin{aligned}
& \sum_{j=1}^{m} \int_{I} y^{j}(t)\left(g^{j}(t, x, \dot{x})+S\left(x \mid C^{j}\right)\right) \mathrm{d} t \\
& \leq \int_{I}\left(\left(\sum_{j=1}^{m} y^{j}(t)\left(g^{j}(t, u, \dot{u})+u(t)^{\mathrm{T}} w^{j}(t)\right)\right)\right. \\
& \left.\quad-\frac{1}{2} p(t)^{\mathrm{T}} G p(t)\right) \mathrm{d} t
\end{aligned}
$$


Using $x(t)^{\mathrm{T}} w^{j}(t) \leq S\left(x(t) / C^{j}\right), t \in I, j=1,2, \cdots, m$, we have,

$$
\begin{aligned}
& \sum_{j=1}^{m} \int_{I} y^{j}(t)\left(g^{j}(t, x, \dot{x})+x(t)^{\mathrm{T}} w^{j}(t)\right) \mathrm{d} t \\
& \leq \int_{I}\left(\left(\sum_{j=1}^{m} y^{j}(t)\left(g^{j}(t, u, \dot{u})+u(t)^{\mathrm{T}} w^{j}(t)\right)\right)\right. \\
& \left.\quad-\frac{1}{2} p(t)^{\mathrm{T}} G p(t)\right) \mathrm{d} t .
\end{aligned}
$$

By the second-order quasi-invexity of

$$
\sum_{j=1}^{m} \int_{I} y^{j}(t)\left(g^{j}(t, . . .)+(.)^{\mathrm{T}} w^{j}(t)\right) \mathrm{d} t,
$$

for $w^{j}(t) \in R^{n}, j=1,2, \cdots, m$ with respect to $\eta$, from this we have,

$$
\begin{aligned}
& \int_{I}\left\{\eta^{\mathrm{T}}\left(\sum_{j=1}^{m} y^{j}(t)\left(g_{u}^{j}+w^{j}(t)\right)\right)\right. \\
& \left.\quad+(D \eta)^{\mathrm{T}}\left(y^{\mathrm{T}}(t) g_{\dot{u}}\right)+\eta^{\mathrm{T}} G p(t)\right\} \mathrm{d} t \leq 0 .
\end{aligned}
$$

By integrating by parts, we have

$$
\begin{aligned}
& \int_{I}\left\{\eta^{\mathrm{T}}\left(\sum_{j=1}^{m} y^{j}(t)\left(g_{u}^{j}+w^{j}(t)\right)\right)\right. \\
& \left.-\eta D\left(y^{\mathrm{T}}(t) g_{\dot{u}}\right)+\eta^{\mathrm{T}} G p(t)\right\} \mathrm{d} t \\
& +\left.\eta(t)^{\mathrm{T}}\left(y^{\mathrm{T}}(t) g_{\dot{u}}\right)\right|_{t=a} ^{t=b} \leq 0 .
\end{aligned}
$$

Using $\eta=0$, at $t=a$ and $t=b$, this yields,

$$
\begin{aligned}
\int_{I} \eta^{\mathrm{T}}\left(\sum_{j=1}^{m} y^{j}(t)\left(g_{u}^{j}+w^{j}(t)\right)\right. \\
\left.\quad-D\left(y(t)^{\mathrm{T}} g_{\dot{u}}\right)+G p(t)\right) \mathrm{d} t \leq 0
\end{aligned}
$$

Using equality constraint (4), we have

$$
\begin{aligned}
0 \leq & \int_{I} \eta^{\mathrm{T}}\left(f_{u}+z(t)-D f_{\dot{u}}+F p(t)\right) \\
= & \int\left(\eta^{\mathrm{T}}\left(f_{u}+z(t)\right)+(D \eta)^{\mathrm{T}} f_{\dot{u}}+\eta^{\mathrm{T}} F p(t)\right) \mathrm{d} t \\
& +\left.\eta^{T} f_{\dot{u}}\right|_{t=a} ^{t}=b, \quad \text { (by integrating by parts) }
\end{aligned}
$$

As earlier, this becomes

$$
\int_{I}\left(\eta^{\mathrm{T}}\left(f_{u}+z(t)\right)+(D \eta)^{\mathrm{T}} f_{\dot{u}}+\eta^{\mathrm{T}} F p(t)\right) \mathrm{d} t \geq 0
$$

This, because of second-order pseudoinvexity of $\int_{I}\left(f(t, . .)+.(.)^{\mathrm{T}} z(t)\right) \mathrm{d} t, z(t) \in R^{n}, t \in I$ with respect to $\eta$, gives

$$
\begin{aligned}
& \int_{I}\left(f(t, x, \dot{x})+x(t)^{\mathrm{T}} z(t)\right) \mathrm{d} t \\
& \geq \int_{I}\left(f(t, u, \dot{u})+u(t)^{\mathrm{T}} z(t)-\frac{1}{2} p(t)^{\mathrm{T}} F p(t)\right) \mathrm{d} t .
\end{aligned}
$$

Since $x(t)^{\mathrm{T}} z(t) \leq S(x(t) / K), t \in I$, we have

$$
\begin{aligned}
& \int_{I}(f(t, x, \dot{x})+S(x(t) / K)) \mathrm{d} t \\
& \geq \int_{I}\left(f(t, u, \dot{u})+u(t)^{\mathrm{T}} z(t)-\frac{1}{2} p(t)^{\mathrm{T}} F p(t)\right) \mathrm{d} t,
\end{aligned}
$$

implying,

$$
\inf (\mathrm{CP}) \geq \sup (\mathrm{M}-\mathrm{WCD}) \text {. }
$$

\section{Theorem 2. (Strong Duality):}

If $\bar{x}(t) \in X$ be an optimal solution of (CP) and is normal, then there exist piecewise smooth functions $y: I \rightarrow R^{m}, \quad z: I \rightarrow R^{n}$ and $w^{j}: I \rightarrow R^{n}$ such that $\left(\bar{x}(t), \bar{y}(t), \bar{z}(t), \bar{w}^{1}(t), \cdots \bar{w}^{m}(t), p(t)=0\right)$ is a feasible solution of (CD) and the two objective values are equal. Furthermore, if the hypothesis of Theorem 1 holds, then $\left(\bar{x}(t), \bar{y}(t), \bar{z}(t), \bar{w}^{1}(t), \cdots, \bar{w}^{m}(t), p(t)\right)$ is an optimal solution of (M-WCD).

Proof: From Lemma 1 there exist piecewise smooth functions $\bar{y}: I \rightarrow R^{m}, \quad \bar{z}: I \rightarrow R^{n}$ and $\quad \bar{w}^{j}: I \rightarrow R^{n}$ $(j=1,2, \cdots, m)$ such that

$$
\begin{gathered}
f_{x}(t, \bar{x}, \dot{\bar{x}})+\bar{z}(t)+\sum_{j=1}^{m} y^{j}(t)\left(g_{x}^{j}(t, \bar{x}, \dot{\bar{x}})+\bar{w}^{j}(t)\right) \\
-D\left(f_{\dot{x}}(t, \bar{x}, \dot{\bar{x}})+\bar{y}^{T}(t) g_{\dot{x}}(t, \bar{x}, \dot{\bar{x}})\right)=0, t \in I \\
\sum_{j=1}^{m} \bar{y}^{j}(t)\left(g^{j}(t, \bar{x}, \dot{\bar{x}})+x(t)^{\mathrm{T}} w^{j}(t)\right)=0, t \in I \\
\bar{x}(t)^{\mathrm{T}} \bar{z}(t)=S(\bar{x}(t) / K), t \in I \\
\bar{x}(t)^{\mathrm{T}} \bar{w}^{j}(t)=S\left(\bar{x}(t) / C^{j}\right), j=1,2, \cdots, m, t \in I \\
\bar{z}(t) \in K, w^{j}(t) \in C^{j}, j=1,2, \cdots, m, t \in I \\
\bar{y}(t) \geq 0, t \in I
\end{gathered}
$$

The above relations imply that $\left(\bar{x}(t), \bar{y}(t), \bar{z}(t), \bar{w}^{1}(t), \cdots, \bar{w}^{m}(t), p(t)=0\right)$ is feasible for (M-WCD).

Also

$$
\begin{aligned}
& \int_{I}(f(t, \bar{x}, \dot{\bar{x}})+S(\bar{x}(t) / K)) \mathrm{d} t \\
& =\int_{I}\left(f(t, \bar{x}, \dot{\bar{x}})+x(t) \bar{z}(t)-\frac{1}{2} p(t)^{\mathrm{T}} F p(t)\right) \mathrm{d} t .
\end{aligned}
$$

This shows the equality of objective functions of the problem. Hence the optimality of $\left(\bar{x}(t), \bar{y}(t), \bar{z}(t), \bar{w}^{1}(t), \cdots, \bar{w}^{m}(t), p(t)\right)$ for (M-WCD) 
follows from weak duality theorem (Theorem1).

Theorem 3 (Strict Converse Duality): Assume that $\left(\mathrm{C}_{1}\right): \int_{I}\left(f(t, . .)+.(.)^{\mathrm{T}} z(t)\right) \mathrm{d} t$ is second-order strictly pseudoinvex and $\int_{I}\left(\sum_{j=1}^{m} y^{j}(t)\left(g^{j}(t, . .)+.(.)^{\mathrm{T}} w^{j}(t)\right)\right)$ is second-order quasi-invex with respect to the same $\eta$.

$\left(\mathrm{C}_{2}\right): \bar{x}(t)$ is an optimal solution for (CP), If $\left(\bar{u}, \bar{y}, \bar{z}, \bar{w}^{1}, \bar{w}^{2}, \cdots, \bar{w}^{m}, p(t)\right)$ is optimal solution of (MWCD), then $\bar{u}(t)=\bar{x}(t), t \in I$.

Proof: We assume that $\bar{u}(t) \neq \bar{x}(t)$ and show that a contradiction follows. Since $\bar{x}(t)$ is an optimal solution of (CP), it follows from Theorem 2, there exist $y: I \rightarrow R^{m}, \quad z: I \rightarrow R^{n}$ and

$w^{j}: I \rightarrow R^{n},(j=1,2, \cdots, m)$ such that

$$
\left(\bar{x}(t), \bar{y}(t), \bar{z}(t), \bar{w}^{1}(t), \bar{w}^{2}(t), \cdots, \bar{w}^{m}(t), p(t)=0\right)
$$

is optimal solution of (M-WCD).

Since

$$
\left(\bar{u}(t), \bar{y}(t), \bar{z}(t), \bar{w}^{1}(t), \bar{w}^{2}(t), \cdots, \bar{w}^{m}(t), p(t)\right)
$$

is an optimal solution of (M-WCD), it follows that

$$
\begin{aligned}
& \int_{I}\left(f(t, \bar{x}, \dot{\bar{x}})+x(t)^{\mathrm{T}} z(t)\right) \mathrm{d} t \\
& =\int_{I}\left(f(t, \bar{u}, \dot{\bar{u}})+\bar{u}(t)^{\mathrm{T}} z(t)-\frac{1}{2} p(t) F p(t)\right) \mathrm{d} t
\end{aligned}
$$

This, because of the second-order strict pseudoinvexity of $\int_{I}\left(f(t, . .)+.(.)^{\mathrm{T}} z(t)\right) \mathrm{d} t$ for all $z(t) \in R^{n}$ gives

$$
\int_{I}\left\{\eta^{\mathrm{T}}\left(f_{u}+z(t)\right)+(D \eta)^{\mathrm{T}} f_{\dot{u}}+\eta^{\mathrm{T}} F p(t)\right\} \mathrm{d} t<0
$$

From the constraint of (CP) and (M-WCD), we have

$$
\begin{aligned}
& \int_{I}\left(\sum_{j=1}^{m} y^{j}(t)\left(g^{j}(t, x, \dot{x})+S\left(x(t) / C^{j}\right)\right)\right) \mathrm{d} t \\
& \leq \int_{I}\left(\sum_{j=1}^{m} y^{j}(t)\left(g^{j}(t, u, \dot{u})+u(t)^{\mathrm{T}} w^{j}(t)\right)\right. \\
& \left.\quad-\frac{1}{2} p(t)^{\mathrm{T}} G p(t)\right) \mathrm{d} t
\end{aligned}
$$

Using $x(t)^{\mathrm{T}} w^{j}(t) \leq S\left(x(t) / C^{j}\right), t \in I, j=1,2, \cdots, m$, from this, we have

$$
\begin{aligned}
& \int_{I}\left(\sum_{j=1}^{m} y^{j}(t)\left(g^{j}(t, x, \dot{x})+x(t)^{\mathrm{T}} w^{j}(t)\right)\right) \mathrm{d} t \\
& \leq \int_{I}\left(\sum_{j=1}^{m} y^{j}(t)\left(g^{j}(t, u, \dot{u})+u(t)^{\mathrm{T}} w^{j}(t)\right)\right. \\
& \left.\quad-\frac{1}{2} p(t)^{\mathrm{T}} G p(t)\right) \mathrm{d} t
\end{aligned}
$$

This, because of $\left(C_{1}\right)$ we have

$$
\begin{aligned}
& \int_{I}\left\{\eta^{\mathrm{T}}\left(\sum_{j=1}^{m} y^{j}(t)\left(g_{u}^{j}(t, u, \dot{u})+w^{j}(t)\right)\right)\right. \\
& \left.+(D \eta)^{\mathrm{T}}\left(y^{\mathrm{T}} g_{\dot{u}}\right)+\eta^{\mathrm{T}}(G p(t))\right\} \mathrm{d} t \leq 0
\end{aligned}
$$

Combining (8) and (9), we have

$$
\begin{aligned}
& 0>\int_{I}\left\{\eta^{\mathrm{T}}\left(f_{u}+z(t)+\sum_{j=1}^{m} y^{j}(t)\left(g_{u}^{j}+w^{j}(t)\right)\right)\right. \\
&\left.+(D \eta)^{\mathrm{T}}\left(f_{\dot{u}}+y(t)^{\mathrm{T}} g_{\dot{u}}\right)+\eta^{\mathrm{T}}(F+G) p(t)\right\} \mathrm{d} t \\
&=\int_{I} \eta^{\mathrm{T}}\left\{\left(f_{u}+z(t)+\sum_{j=1}^{m} y^{j}(t)\left(g_{u}^{j}+w^{j}(t)\right)\right)\right. \\
&\left.-D\left(f_{\dot{u}}+y(t)^{\mathrm{T}} g_{\dot{u}}\right)+(F+G) p(t)\right\} \mathrm{d} t \\
&+\left.\eta^{\mathrm{T}}\left(f_{\dot{u}}+y(t)^{\mathrm{T}} g_{\dot{u}}\right)\right|_{t=a} ^{t=b} .
\end{aligned}
$$

Using $\eta=0$, at $t=a$ and $t=b$, this implies

$$
\begin{aligned}
\int_{I} \eta^{T}\{ & \left\{\left(f_{u}+z(t)+\sum_{j=}^{m} y^{j}(t)\left(g_{u}^{j}+w^{j}(t)\right)\right)\right. \\
& \left.-D\left(f_{\dot{u}}+y(t)^{\mathrm{T}} g_{\dot{u}}\right)+(F+G) p(t)\right\} \mathrm{d} t<0,
\end{aligned}
$$

contradicting the equality constraint of (M-WCD), hence $\bar{u}(t)=\bar{x}(t), t \in I$.

Theorem 4. (Converse Duality): Assume that

$\left(\mathrm{H}_{1}\right):\left(x(t), y(t), z(t), w^{1}(t), w^{2}(t), \cdots, w^{m}(t), p(t)\right)$ is an optimal solution of (M-WCD).

$\left(\mathrm{H}_{2}\right)$ : The vectors $\left\{F^{i}, G^{i}, i=1,2, \cdots, m\right\}$ are linear independent where $F^{i}$ and $G^{i}$ are the $i^{\text {th }}$ row of $F$ and $G$ respectively, and

$\left(\mathrm{H}_{3}\right)$ :

$$
\sum_{t \in I} y^{j}(t)\left(g_{x}^{j}+w^{j}(t)\right)-D\left(y(t)^{\mathrm{T}} g_{\dot{x}}\right)+G(t) p(t) \neq 0,
$$

and

$$
\left(\mathrm{H}_{4}\right) \text { : either } \int_{I} p(t)^{\mathrm{T}}\left(G+y(t)^{\mathrm{T}} g_{x x}\right) p(t) \mathrm{d} t>0
$$

and $\int_{I} p(t)^{\mathrm{T}} \sum_{j=1}^{m} y^{j}(t)\left(g_{x}^{j}+w^{j}(t)\right) \mathrm{d} t \geq 0$

or $\int_{I} p(t)^{\mathrm{T}}\left(G+y(t)^{\mathrm{T}} g_{x x}\right) p(t) \mathrm{d} t<0$

and $\int_{I} p(t)^{\mathrm{T}} \sum_{j=1}^{m} y^{j}(t)\left(g_{x}^{j}+w^{j}(t)\right) \mathrm{d} t \leq 0$

Then $\bar{x}(t)$ is feasible for (CP) and the two objective functionals have the same value. Also, if Theorem 1 holds for all feasible solution of (CP) and (M-WCD), then $\bar{x}(t)$ is an optimal solution of (CP). 


\section{Proof: Since}

$\left(x(t), y(t), z(t), w^{1}(t), \cdots, w^{m}(t), p(t)\right)$ is an optimal solution of (M-WCD), by results of Schester [10], there exists $\tau \in R, \gamma \in R$ and piecewise smooth function $\theta: I \rightarrow R^{n}$ and $\eta: I \rightarrow R^{m}$ such that following Fritz John optimality conditions are satisfied:

$$
\begin{aligned}
& \tau\left[\left(f_{x}+z(t)-D f_{\dot{x}}\right)-\frac{1}{2}\left(p(t)^{\mathrm{T}} F p(t)\right)_{x}\right. \\
& +\frac{1}{2} D\left(p(t)^{\mathrm{T}} F p(t)\right)_{\dot{x}}-\frac{1}{2} D^{2}\left(p(t)^{\mathrm{T}} F p(t)\right)_{\ddot{x}} \\
& \left.+\frac{1}{2} D^{3}\left(p(t)^{\mathrm{T}} F p(t)\right)_{\dddot{x}}-\frac{1}{2} D^{4}\left(p(t)^{\mathrm{T}} F p(t)\right)_{\bar{x}}\right] \\
& +\theta(t)^{\mathrm{T}}\left[f_{x x}-D f_{\dot{x} x}+\left(y(t)^{\mathrm{T}} g_{x}\right)_{x}-D\left(y(t)^{\mathrm{T}} g_{x}\right)_{\dot{x}}\right. \\
& -D\left(f_{\dot{x} x}+\left(y(t)^{\mathrm{T}} g_{\dot{x}}\right)_{x}\right)+D\left(D\left(f_{\dot{x} \dot{x}}-\left(y(t)^{\mathrm{T}} g_{\dot{x}}\right)_{\dot{x}}\right)\right) \\
& -D^{2}\left(D f_{\ddot{x} \ddot{x}}-\left(y(t)^{\mathrm{T}} g_{\dot{x}}\right)_{\ddot{x}}\right)+((F+G) p(t))_{x} \\
& -D((F+G) p(t))_{\dot{x}}+D^{2}((F+G) p(t))_{\ddot{x}} \\
& \left.-D^{3}((F+G) p(t))_{\ddot{x}}+D^{4}((F+G) p(t))_{\ddot{x}}\right] \\
& +\gamma\left[\sum_{j=1}^{m} y^{j}(t)\left(g_{x}^{j}+w^{j}\right)-D\left(y^{\mathrm{T}}(t) g_{\dot{x}}\right)\right. \\
& -\frac{1}{2}\left(p(t)^{\mathrm{T}} G p(t)\right)_{x}+\frac{1}{2} D\left(p(t)^{\mathrm{T}} G p(t)\right)_{\dot{x}} \\
& -\frac{1}{2} D^{2}\left(p(t)^{\mathrm{T}} G p(t)\right)_{\dddot{X}}+\frac{1}{2} D^{3}\left(p(t)^{\mathrm{T}} G p(t)\right)_{\dddot{X}} \\
& \left.-\frac{1}{2} D^{4}\left(p(t)^{\mathrm{T}} G p(t)\right)_{x}\right]=0 \text {, } \\
& \theta(t)^{\mathrm{T}}\left[\left(g_{x}^{j}+w^{j}(t)\right)+g_{x x}^{j} p(t)\right] \\
& +\gamma\left(\left(g^{j}+x(t)^{\mathrm{T}} w^{j}(t)\right)-\frac{1}{2} p(t)^{\mathrm{T}} g_{x x}^{j} p(t)\right) \\
& +\eta^{j}(t)=0, t \in I \\
& (\theta(t)+\tau p(t)) F+(\theta(t)+\gamma p(t)) G=0, t \in I \\
& \tau x(t)+\theta(t) \in N_{k}(z(t))
\end{aligned}
$$

$$
\begin{gathered}
\theta(t) y^{j}(t)+\gamma x(t) \in N_{C_{j}}\left(w^{j}(t)\right) \\
\gamma \int_{I}\left(\sum_{i \in I} y^{j}(t)\left(g^{j}+x(t)^{\mathrm{T}} w^{j}(t)\right)-\frac{1}{2} p(t)^{\mathrm{T}} G p(t)\right)=0 \\
\eta^{\mathrm{T}}(t) y(t)=0, t \in I \\
(\tau, \eta(t), \gamma) \geq 0, t \in I \\
(\tau, \eta(t), \theta(t), \gamma) \neq 0, t \in I
\end{gathered}
$$

Using the hypothesis $\left(\mathrm{H}_{2}\right)$ in (12), we have

$$
\begin{aligned}
& \theta(t)+\tau p(t)=0, t \in I \\
& \theta(t)+\gamma p(t)=0, t \in I
\end{aligned}
$$

Using (4), (19) and (20) in (10), we have (see (21) below) Let $\gamma=0$, then (20) gives $\theta(t)=0, t \in I$, (19) implies $\tau p(t)=0, t \in I$.

Consequently from (21), we have

$$
\begin{gathered}
\tau\left(\sum_{j=1}^{m} y^{j}(t)\left(g_{x}^{j}(t, \bar{x}, \dot{\bar{x}})+w^{j}(t)\right)\right. \\
\left.-D\left(y(t)^{\mathrm{T}} g_{\dot{x}}\right)+G p(t)\right)=0, t \in I
\end{gathered}
$$

By the hypothesis $\left(\mathrm{H}_{3}\right)$, this implies $\tau=0$.

The relation (11) implies $\eta(t)=0, t \in I$.

Hence $(\tau, \theta(t), \eta(t), \gamma)=0$ contradicting the Fritz John condition, Hence $\gamma>0, \& \tau>0$.

Pre-multiplying (11) by $y^{j}(t)$ and Using (16), we have

$$
\begin{gathered}
\theta(t)^{\mathrm{T}}\left[\sum_{j=1}^{m} y^{j}(t)\left(g^{j}+w^{j}(t)\right)+\left(y(t)^{\mathrm{T}} g_{x x}\right) p(t)\right] \\
-\gamma\left(\sum_{j=1}^{m} y^{j}(t)\left(g^{j}+x(t)^{\mathrm{T}} w^{j}(t)\right)\right. \\
\left.-\frac{1}{2} p(t)^{\mathrm{T}}\left(y(t)^{\mathrm{T}} g_{x x}\right) p(t)\right)=0 .
\end{gathered}
$$

Integrating and then using (15), we have

$$
\begin{aligned}
& (\tau-\gamma)\left[\sum_{j=1}^{m} y^{j}(t)\left(g_{x}^{j}+w^{j}(t)\right)-D\left(y(t)^{\mathrm{T}} g_{\dot{x}}\right)+G p(t)\right]+\frac{\tau}{2}\left[\left(p(t)^{T} F p(t)\right)-D\left(p(t)^{\mathrm{T}} F p(t)\right)_{\dot{x}}+D^{2}\left(p(t)^{\mathrm{T}} F p(t)\right)_{\ddot{x}}\right. \\
& \left.-D^{3}\left(p(t)^{\mathrm{T}} F p(t)\right)_{\ddot{x}}+D^{4}\left(p(t)^{T} F p(t)\right)_{\ddot{x}}\right]+\theta(t)^{\mathrm{T}}\left[((F+G) p(t))_{x}-D((F+G) p(t))_{\dot{x}}+D^{2}((F+G) p(t))_{\ddot{x}}\right. \\
& \left.-D^{3}((F+G) p(t))_{\ddot{x}}+D^{4}((F+G) p(t))_{\ddot{x}}\right]+\frac{\gamma}{2}\left[\left(p(t)^{\mathrm{T}} G p(t)\right)_{x}-D\left(p(t)^{\mathrm{T}} G p(t)\right)_{\dot{x}}+D^{2}\left(p(t)^{\mathrm{T}} G p(t)\right)_{\ddot{x}}\right. \\
& \left.-D^{3}\left(p(t)^{\mathrm{T}} G p(t)\right)_{\ddot{x}}+D^{4}\left(p(t)^{\mathrm{T}} G p(t)\right)_{\ddot{x}}\right]=0, \quad t \in I
\end{aligned}
$$




$$
\begin{aligned}
& \int_{I} \theta(t)^{\mathrm{T}}\left[\sum_{j=1}^{m} y^{j}(t)\left(g_{x}^{j}+w^{j}(t)\right)+\left(y(t)^{\mathrm{T}} g_{x x}\right) p(t)\right] \mathrm{d} t \\
& -\frac{\gamma}{2}\left(\iint_{I}\left(p(t)^{\mathrm{T}} G p(t)-p(t)^{\mathrm{T}}\left(y(t)^{\mathrm{T}} g_{x x}\right) p(t)\right)\right) \mathrm{d} t=0
\end{aligned}
$$

Putting $\theta(t)=-\gamma p(t)$ we have

$$
\begin{aligned}
& -\gamma \int_{I} p(t)^{\mathrm{T}}\left(\sum_{j=1}^{m} y^{j}(t)\left(g_{x}^{j}+w^{j}(t)\right)\right) \mathrm{d} t \\
& -\frac{\gamma}{2} \int_{I} p(t)^{\mathrm{T}}\left(G+y(t)^{\mathrm{T}} g_{x x}\right) p(t) \mathrm{d} t=0
\end{aligned}
$$

gives

$$
\begin{aligned}
& 2 \int_{I} p(t)^{\mathrm{T}}\left(\sum_{j=1}^{m} y^{j}(t)\left(g_{x}^{j}+w^{j}(t)\right)\right) \mathrm{d} t \\
& +\int_{I} p(t)^{\mathrm{T}}\left(G+y(t)^{\mathrm{T}} g_{x x}\right) p(t) \mathrm{d} t=0
\end{aligned}
$$

This, in view of the hypothesis $\left(\mathrm{H}_{4}\right)$ yields, $p(t)=0, t \in I$ we have $\bar{x}(t) \in N_{k}(z(t))$ and $\bar{x}(t) \in N_{C^{j}}\left(\bar{w}^{j}(t)\right), j=1,2, \cdots, m$.

These respectively imply $x(t)^{\mathrm{T}} z(t)=S(x(t) / K)$ and $x(t)^{\mathrm{T}} w^{j}(t)=S\left(x(t) / C^{j}\right), j=1,2, \cdots, m$.

Multiplying the relation (11) by $y^{j}(t)$ and using (16) along with $p(t)=0, t \in I$, we have

$$
\sum_{j=1}^{m} y^{j}(t)\left(g^{j}+\bar{x}(t)^{\mathrm{T}} w^{j}(t)\right)=0, t \in I
$$

and also $g^{j}(t, \bar{x}, \dot{\bar{x}})+S\left(x(t) / C^{j}\right) \leq 0, t \in I$ implying the feasibility of $\bar{x}(t)$ for (CP).

Finally,

$$
\begin{aligned}
& \int_{I}(f(t, \bar{x}, \dot{\bar{x}})+S(\bar{x}(t) / K)) \mathrm{d} t \\
& =\int_{I}\left(f(t, \bar{x}, \dot{\bar{x}})+\bar{x}(t)^{\mathrm{T}} z(t)-\frac{1}{2} p(t)^{\mathrm{T}} F p(t)\right) \mathrm{d} t
\end{aligned}
$$

By Theorem 1, it implies that $\bar{x}(t)$ is an optimal solution of (CP).

\section{Special Cases}

Let for $t \in I, \quad A(t), B^{j}(t), j=1,2, \cdots, m$, be positive semidefinite matrices and continuous on $I$.

Then $\left(x(t)^{\mathrm{T}} A(t) x(t)\right)^{1 / 2}=S(x(t) / K), t \in I$, where

$$
K=\left\{A(t) z(t) \mid z(t)^{\mathrm{T}} A(t) z(t) \leq 1, t \in I\right\}
$$$$
\left(x(t)^{\mathrm{T}} B^{j}(t) x(t)\right)^{1 / 2}=S\left(x(t) / C^{j}\right), j=1,2, \cdots, m, t \in I .
$$

Replacing $S(x(t) / K)$ by $\left(x(t)^{\mathrm{T}} A(t) x(t)\right)^{1 / 2}$ and $S\left(x(t) / C^{j}\right)$, by
$\left(x(t)^{\mathrm{T}} B^{j}(t) x(t)\right)^{1 / 2}, j=1,2, \cdots, m, t \in I$.

We have the following problems:

$\left(\mathrm{CP}_{2}\right)$ : Minimize $\int_{I}\left\{f(t, x, \dot{x})+\left(x(t)^{\mathrm{T}} A(t) x(t)\right)^{1 / 2}\right\} \mathrm{d} t$

subject to

$$
\begin{gathered}
x(a)=0=x(b), \\
g^{j}(t, x, \dot{x})+\left(x(t)^{\mathrm{T}} B^{j}(t) x(t)\right)^{1 / 2} \leq 0, \\
t \in I, j=1,2, \cdots, m .
\end{gathered}
$$

\section{(M-WCD $)^{2}$ :}

$$
\begin{gathered}
\text { Maximize } \int_{I}\left(f(t, u, \dot{u})+u(t)^{\mathrm{T}} A(t) z(t)\right. \\
\left.-\frac{1}{2} p(t)^{\mathrm{T}} F P(t)\right) \mathrm{d} t
\end{gathered}
$$

subject to

$$
\begin{aligned}
& \qquad u(a)=0=u(b), \\
& f_{u}(t, u, \dot{u})+A(t) z(t) \\
& +\sum_{j=1}^{m} y^{j}(t)\left(g_{u}^{j}(t, u, \dot{u})+B^{j}(t) w^{j}(t)\right) \\
& -D\left(f_{\dot{u}}(t, u, \dot{u})+y(t)^{\mathrm{T}} g_{\dot{u}}(t, u, \dot{u})\right)+(F+G) p(t)=0, \\
& t \in I
\end{aligned}
$$

$$
\begin{gathered}
\int_{I}\left(\sum_{j=1}^{m} y^{j}(t)\left(g^{j}+u(t)^{\mathrm{T}} B^{j}(t) \omega^{j}(t)\right)\right. \\
\left.-\frac{1}{2} p^{\mathrm{T}}(t) G p(t)\right) \mathrm{d} t \geq 0 \\
z(t)^{\mathrm{T}} A(t) z(t) \leq 1, \quad t \in I \\
w^{j}(t) B^{j}(t) w^{j}(t) \leq 1, t \in I, j=1,2, \cdots, m
\end{gathered}
$$

If $\left(x(t)^{\mathrm{T}} B^{j}(t) x(t)\right)^{1 / 2}, j=1,2, \cdots, m$ are suppressed from the constraints of $\left(\mathrm{CP}_{2}\right)$, we have the following problem studied for duality by Husain and Srivastava [4].

$$
\left(\mathrm{CP}_{3}\right) \text { : Minimize } \int_{I}\left(f(t, x, \dot{x})+\left(x(t)^{\mathrm{T}} A(t) x(t)\right)^{1 / 2}\right) \mathrm{d} t
$$

subject to

$$
\begin{gathered}
x(a)=0=x(b) \\
g(t, x, \dot{x}) \leq 0, t \in I .
\end{gathered}
$$

$\left(\mathrm{M}-\mathrm{WCD}_{3}\right)$ : 


$$
\begin{gathered}
\text { Maximize } \int_{I}\left(f(t, u, \dot{u})+u(t)^{\mathrm{T}} A(t) z(t)\right. \\
\left.-\frac{1}{2} p(t)^{\mathrm{T}} F P(t)\right) \mathrm{d} t \\
u(a)=0=u(b) \\
f_{u}(t, u, \dot{u})+A(t) z(t)+y(t)^{\mathrm{T}} g_{u}(t, u, \dot{u}) \\
-D\left(f_{\dot{u}}(t, u, \dot{u})+y(t)^{\mathrm{T}} g_{\dot{u}}(t, u, \dot{u})\right) \\
+(F+G) p(t)=0, t \in I \\
\int_{I}\left(y(t)^{\mathrm{T}} g(t, u, \dot{u})-\frac{1}{2} p(t)^{\mathrm{T}} G p(t)\right) \mathrm{d} t \geq 0, t \in I \\
z(t)^{\mathrm{T}} A(t) z(t) \leq 1, t \in I \\
y(t) \geq 0, t \in I .
\end{gathered}
$$

\section{Problems with Natural Boundary Values}

In this section, we formulate a pair of nondifferentiable dual variational problems with natural boundary values rather than fixed end points.

$\left(\mathrm{CP}_{0}\right)$ : Minimize $\int\{f(t, x, \dot{x})+S(x(t) \mid K)\} \mathrm{d} t$ subject to $x(a)=0 \pm x(b)$,

$$
\begin{gathered}
g^{j}(t, x, \dot{x})+S\left(x(t) \mid C^{j}\right) \leq 0, t \in I, j=1,2 \cdots, m \\
\left(M-W C D_{0}\right): \\
\text { Maximize } \int_{I}\left\{f(t, u, \dot{u})+u(t)^{\mathrm{T}} z(t)\right. \\
\left.-\frac{1}{2} p(t)^{\mathrm{T}} F(t) p(t)\right\} \mathrm{d} t
\end{gathered}
$$

subject to

$$
\begin{gathered}
u(a)=0=u(b) \\
f_{u}(t, u, \dot{u})+z(t)^{\mathrm{T}}+\sum_{j=1}^{m} y^{j}(t)\left(g_{u}^{j}(t, u, \dot{u})+w^{j}(t)\right) \\
-D\left(f_{\dot{u}}(t, u, \dot{u})+y(t)^{\mathrm{T}} g_{\dot{u}}(t, u, \dot{u})\right) \\
+(F+G) p(t)=0, t \in I \\
\int_{I}\left\{\sum_{j=1}^{m} y^{j}(t)\left(g^{j}(t, u, \dot{u})+u(t)^{\mathrm{T}} w^{j}(t)\right)\right. \\
\left.-\frac{1}{2} p(t)^{\mathrm{T}} G p(t)\right\} \mathrm{d} t \geq 0, t \in I \\
y(t) \geq 0, t \in I \\
z(t) \in K, w^{j}(t) \in C^{j}, j=1,2, \cdots, m, t \in I
\end{gathered}
$$

$$
\begin{gathered}
f_{\dot{u}}(t, u, \dot{u})=0 \text { at } t=a \text { and } t=b, \\
y(t)^{\mathrm{T}} g_{\dot{u}}(t, u, \dot{u})=0 \text {, at } t=a \text { and } t=b .
\end{gathered}
$$

\section{Nonlinear Programming Problems}

If all functions in the problems $\left(\mathrm{CP}_{0}\right)$ and $\left(\mathrm{M}-\mathrm{WCD}_{0}\right)$ are independent of $t$, then these problems will reduce to the following nonlinear programming problems studied by Husain et al. [7].

$\left(\mathrm{CP}_{1}\right)$ : Minimize $f(x)+S(x \mid K)$

subject to

$$
\begin{aligned}
& g^{j}(x)+S\left(x \mid C^{j}\right) \leq 0, j=1,2, \cdots, m . \\
& \left(\mathrm{CD}_{1}\right) \text { : Maximize } f(u)+u^{\mathrm{T}} z-\frac{1}{2} p^{\mathrm{T}} F p
\end{aligned}
$$
subject to

$$
\begin{gathered}
f_{u}(u)+z+\sum_{j=1}^{m} y^{j}\left(g_{u}^{j}(u)+w^{j}\right)+(F+G) p=0 \\
\left(\sum_{j=1}^{m} y^{j}\left(g^{j}+u^{\mathrm{T}} w^{j}\right)-\frac{1}{2} p^{\mathrm{T}} G p\right) \geq 0, \\
z \in K, w^{j} \in C^{j}, j=1,2, \cdots, m,
\end{gathered}
$$

where $F=f_{u u}(u)$ and $G=y^{\mathrm{T}} g_{u u}(u)$.

\section{REFERENCES}

[1] X. H. Chen, "Second Order Duality for the Variational Problems," Journal of Mathematical Analysis and Applications, Vol. 286, No. 1, 2003, pp. 261-270. http://dx.doi.org/10.1016/S0022-247X(03)00481-5

[2] I. Husain, A. Ahmed and M. Masoodi, "Second Order Duality for Variational Problems," European Journal of Pure and Applied Mathematics, Vol. 2, No. 2, 2009, pp. 278-295.

[3] I. Husain and M. Masoodi, "Second-Order Duality for a Class of Nondifferentiable Continuous Programming Problems," European Journal of Pure \& Applied Mathematics, Vol. 5, No. 3, 2012, pp. 390-400.

[4] I. Husain and S. K. Shrivastav, "On Second-Order Duality in Nondifferentiable Continuous Programming," American Journal of Operations Research, Vol. 2, No. 3, 2012, pp. 289-295.

http://dx.doi.org/10.4236/ajor.2012.23035

[5] O. L. Mangasarian, "Second and Higher Order Duality in Nonlinear Programming,” Journal of Mathematical Analysis and Applications, Vol. 51, No. 3, 1979, pp. 605-620. http://dx.doi.org/10.1016/0022-247X(75)90111-0

[6] I. Husain and M. Masoodi "Second-Order Duality for Continuous Programming Containing support Functions," Applied Mathematics, Vol. 1, No. 6, 2010, pp. 534-541. http://dx.doi.org/10.4236/am.2010.16071

[7] I. Husain, A. Ahmed and M. Masoodi, "Second Order Duality in Mathematical Programming with Support Functions," Journal of Informatics and Mathematical Sciences, Vol. 1, No. 2-3, 2009, pp. 165-182. 
[8] I. Husain and Z. Jabeen, "Continuous Programming Containing Support Functions,” Journal of Applied Mathematics \& Informatics, Vol. 26, No. 1-2, 2008, pp. 75-106.

[9] B. Mond and M. Schechter, "Nondifferentiable Symmetric Duality," Bulletin of the Australian Mathematical Society, Vol. 53, 1996, pp. 177-188.

\section{http://dx.doi.org/10.1017/S0004972700016890}

[10] M. Schechter, “More on Subgradient Duality,” Journal of Mathematical Analysis and Applications, Vol. 71, No. 1, 1979, pp. 251-262.

http://dx.doi.org/10.1016/0022-247X(79)90228-2 